\title{
Rapid Clinical and Radiographic Response With Combined Dabrafenib and Trametinib in Adults With BRAF-Mutated High-Grade Glioma
}

\author{
Tanner M. Johanns, MD, PhD ${ }^{\mathrm{a}, \mathrm{b}}$; Cole J. Ferguson, MD, PhDc; Patrick M. Grierson, MD, PhDª, \\ Sonika Dahiya, MD ${ }^{\mathrm{b}, \mathrm{c}}$; and George Ansstas, MD $\mathrm{a,b}$
}

\begin{abstract}
BRAF V600E mutations have been successfully treated with targeted therapy in melanoma, non-small cell lung cancer, and thyroid cancer. Interestingly, these mutations have also been identified in a subset of pediatric and adult brain tumors, with several cases reportedly responding to targeted therapy. However, these reports have been limited to single-agent BRAF inhibitor therapy and recurrent disease. Herein, we report dramatic clinical and radiographic responses to combination dabrafenib (BRAF inhibitor) and trametinib (MEK inhibitor) in 2 adults with high-grade gliomas (HGGs), with 1 patient treated in the first-line setting. These observations, together with prior case reports, advocate for routine screening of BRAF point mutations in adult HGGs, and suggest that treatment with dual-targeted therapy, even in newly diagnosed cases, is safe and effective.
\end{abstract}

J Natl Compr Canc Netw 2018;16(1):4-10 doi: 10.6004/jnccn.2017.7032

The activating BRAF V600E mutation has been successfully targeted with dual molecular therapy in melanoma, non-small cell lung carcinoma (NSCLC), and thyroid cancer. BRAF V600E mutations have also been identified in a subset of patients with pediatric and adult brain tumors, and several case reports have indicated the potential efficacy of targeted therapy in these patients. To date, these studies have mostly been performed using single-agent BRAF inhibitor therapy in recurrent lowgrade gliomas (LGGs; Table 1). This report describes the use of combination dabrafenib (BRAF inhibitor) and trametinib (MEK inhibitor) in 2 adults with high-grade gliomas (HGGs). The first patient was treated at time of

From a Department of Medicine, Division of Medical Oncology, Washington University School of Medicine; 'biteman Cancer Center at Barnes-Jewish Hospital and Washington University School of Medicine; 'Division of Neuropathology, Department of Pathology and Immunology, Washington University School of Medicine, St. Louis, Missouri.

Submitted May 18, 2017; accepted for publication September 5, 2017.

Dr. Ansstas has disclosed that he is a member of the advisory board and speaker's bureau for Novocure. The remaining authors have disclosed that they have no financial interests, arrangements, affiliations, or commercial interests with the manufacturers of any products discussed in this article or their competitors. diagnosis, whereas the second was treated at time of recurrence in conjunction with the antiangiogenic agent bevacizumab. Both patients showed rapid and dramatic clinical and radiographic responses.

\section{Case Reports}

\section{Patient 1}

Patient 1 was a previously healthy 28-year-old female who developed generalized tonic-clonic seizures and was ultimately found to have a left hippocampal lesion. The seizures initially began when the patient was 5 weeks pregnant; however, a noncontrast head CT at the time was unremarkable. A postpartum brain T2-weighted,

Drs. Johanns and Grierson are supported by the NIH T32 HL007088 training grant through the Division of Hematology and Physician-Scientist Training Program at Washington University School of Medicine.

Correspondence: George Ansstas, MD, Division of Medical Oncology, Department of Medicine, Washington University School of Medicine, 660 South Euclid Avenue, St. Louis, MO 63110.

E-mail: gansstas@wustl.edu; and

Sonika Dahiya, MD, Division of Neuropathology, Department of Pathology and Immunology, Washington University School of Medicine, Campus Box 8118, 660 South Euclid Avenue, St Louis, MO 63110.

E-mail: sdahiya@wustl.edu 
Targeted Therapy in BRAF-Mutated GBM

Table 1. Studies Using Single-Agent BRAF Inhibitors in Recurrent Low-Grade Gliomas

\begin{tabular}{|c|c|c|c|c|c|}
\hline Age/Sex & Diagnosis & $\begin{array}{l}\text { BRAF } \\
\text { Inhibitor }\end{array}$ & Response & $\begin{array}{l}\text { Duration of } \\
\text { Response }\end{array}$ & Reference \\
\hline \multicolumn{6}{|l|}{ Pediatric } \\
\hline $2 \mathrm{mo}, \mathrm{F}$ & Hypothalamic-chiasmatic glioma & Dabrafenib & PR & $>10 \mathrm{mo}^{\mathrm{a}}$ & 40 \\
\hline $18 \mathrm{mo}, \mathrm{F}$ & Peduncular anaplastic ganglioglioma & Vemurafenib & PR & $>20 \mathrm{mo}^{\mathrm{a}}$ & 33 \\
\hline $23 \mathrm{mo}, \mathrm{M}$ & Pilomyxoid astrocytoma & Vemurafenib & PR & $>15 \mathrm{mo}^{\mathrm{a}}$ & 36 \\
\hline $28 \mathrm{mo}, \mathrm{M}$ & Brainstem ganglioglioma & Vemurafenib & PR & $>6 \mathrm{mo}^{\mathrm{a}}$ & 39 \\
\hline $6 y, M$ & Thalamic anaplastic ganglioglioma & Vemurafenib & PR & $3 \mathrm{mo}$ & 33 \\
\hline $8 y, M$ & Brainstem ganglioglioma & Vemurafenib & PR & $>14 \mathrm{mo}^{\mathrm{a}}$ & 38 \\
\hline $9 y, M$ & Glioblastoma & Vemurafenib & CR & $>6 \mathrm{mo}^{\mathrm{a}}$ & 16 \\
\hline $13 y, F$ & Brainstem ganglioglioma & Vemurafenib & PR & $>12 w^{a}$ & 34 \\
\hline \multicolumn{6}{|l|}{ Adult } \\
\hline $25 y, M$ & Anaplastic ganglioglioma & Dabrafenib & CR & $>24 \mathrm{mo}^{\mathrm{a}}$ & 41 \\
\hline 34 y, F & PXA & Vemurafenib & SD & $4 \mathrm{mo}$ & 28 \\
\hline $35 y, F$ & Anaplastic PXA & Dabrafenib & PR & $3 \mathrm{mo}$ & 35 \\
\hline $41 \mathrm{y}, \mathrm{M}$ & Anaplastic PXA & Vemurafenib & CR & $>12 w^{a}$ & 42 \\
\hline $43 \mathrm{y}, \mathrm{M}$ & Anaplastic PXA & Vemurafenib & PD & NR & 28 \\
\hline $47 \mathrm{y}, \mathrm{F}$ & PXA & Vemurafenib & SD & $6 \mathrm{mo}$ & 28 \\
\hline $53 \mathrm{y}, \mathrm{M}$ & PXA & Vemurafenib & PR & $10 \mathrm{mo}$ & 28 \\
\hline
\end{tabular}

Abbreviations: $C R$, complete response; $F$, female; $M$, male; NR, not reported; $P D$, progressive disease; PR, partial response; PXA, pleomorphic xanthoastrocytoma; SD, stable disease.

${ }^{a}$ Duration of response at time of publication.

fluid-attenuated inversion recovery (T2/FLAIR) MRI performed 9 months later revealed an illdefined hyperintense lesion with central contrast enhancement within the left hippocampal body extending into the hippocampal tail, considered to be most consistent with a LGG. A repeat MRI 3 months later demonstrated an increase in size of the lesion with local extension into the basal ganglia, anterior temporal lobe, and midbrain. Given the rate of progression, a left temporal craniotomy was performed, with a subtotal resection achieved. The postoperative course was complicated by rightsided hemiparesis and word-finding difficulties due to a subdural hematoma requiring evacuation and obstructive hydrocephalus requiring shunt placement.

Histopathologic evaluation of the resected tumor showed a diffusely infiltrative glioma with moderate to dense cellularity, marked pleomorphism, brisk mitotic activity, and predominantly epithelioid morphology (Figure 1A). Although necrosis was not convincingly present, large areas of hemorrhage, focal endothelial hyperplasia (Figure 1B) and vascular thrombosis were present. The tumor was widely infiltrative (Figure 1C). Fluorescence in situ hybridization (FISH) showed polysomy of chromosome 7 al- beit without EGFR amplification, and there was no PTEN (or monosomy 10q) loss. A targeted gene sequencing panel (FoundationOne, Foundation Medicine, Cambridge, MA) revealed loss of CDKN2A/B and TERT promoter mutations. Interestingly, gene sequencing also revealed the presence of a BRAF V600E mutation, which was corroborated by immunohistochemistry (Figure 1D). No mutations in IDH1 or IDH2 were identified through sequencing, and immunohistochemistry showed retained ATRX and INI1 expression. MGMT was unmethylated. Brisk proliferation was evident on $\mathrm{Ki}-67$, particularly in the epithelioid regions. No areas reminiscent of classic pleomorphic xanthoastrocytoma (PXA) were identified in this tumor. Specifically, there was lack of eosinophilic granular bodies and reticulin-rich areas.

The collective findings of diffuse growth pattern, largely epithelioid histology, marked anaplasia, and lack of classic PXA phenotype, in conjunction with polysomy of chromosome 7 and TERT promoter mutations, were favored to represent epithelioid glioblastoma (GBM).

A brain MRI performed 4 weeks postoperatively demonstrated interval increased involvement of 

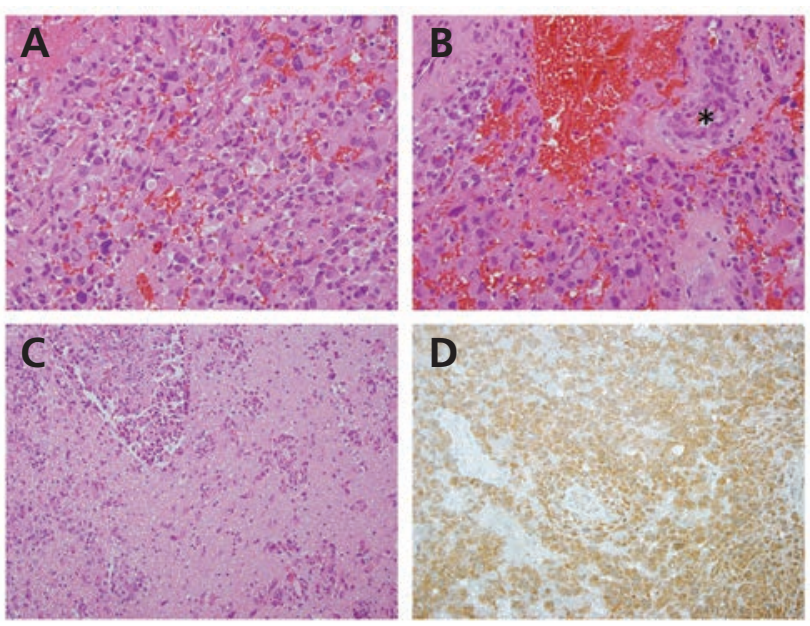

Figure 1. Histopathology of tumor from patient 1, composed of mostly epithelioid glial cells ( $\mathrm{A}$; hematoxylin-eosin [HE] staining, original mag-nification $\times 20$ ) with focal endothelial hyperplasia (B, asterisk; $\mathrm{HE}$ staining, original magnification $\times 20$ ), prominent infiltrative growth pattern ( $\mathrm{C}$; HE staining, original magnification $\times 10)$, and diffuse cytoplasmic immunoreactivity for mutant BRAF V600E ( $\mathrm{D}$; original magnification $\times 20$ ).

the anterior left temporal lobe and thalamus (Figure $2 \mathrm{~A}$, black arrow), with residual disease enhancement extending from the resection cavity into the cerebral peduncle inferiorly, as well as interval development of enhancement of cranial nerves V, VII, and VIII, along with the optic chiasm and pituitary stalk, and new nodular foci of enhancement along the temporal dura (Figure 2A, white arrow). Overall, these findings were consistent with leptomeningeal gliomatosis. Despite the concern for continued progression in the absence of aggressive therapy, all adjuvant treatment was deferred due to persistent

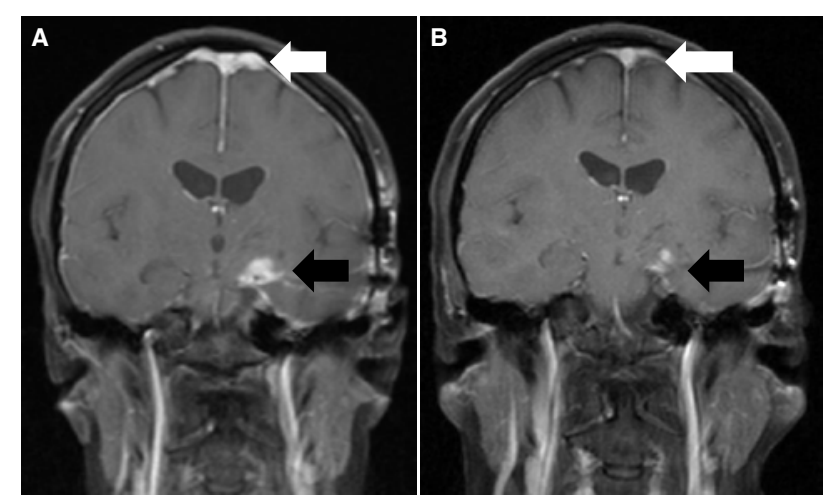

Figure 2. Brain MRI of patient 1 before (A) and 4 weeks after (B) treatment with dabrafenib and trametinib. Images are representative coronal views from the postcontrast T2-weighted, fluid-attenuated inversion recovery (FLAIR) sequences, which demonstrate contrastenhancing lesions in the thalamic region (black arrow) and temporal dura (white arrow), consistent with residual disease postresection and new metastatic foci of disease, respectively. poor functional status. Unfortunately, 4 months after surgery the patient continued to have persistent poor functional status, precluding the possibility of even radiation monotherapy, so the decision was made to begin dabrafenib (150 mg, twice daily) and trametinib (2 mg, once daily). Remarkably, within 1 week of initiating dual-targeted therapy, the patient experienced a dramatic improvement in speech, overall strength, and endurance, although she continued to have right-sided hemiparesis. Four weeks later, repeat brain MRI revealed near complete resolution of the leptomeningeal gliomatosis with no evidence of intraparenchymal disease progression, although there was a persistent T2/FLAIR signal surrounding the resection cavity (Figure 2B). Over the subsequent months, the patient experienced gradual improvement in right-sided hemiparesis while experiencing no significant side effects from the combination therapy.

Unfortunately, 11 months after initiating treatment, MRI showed disease progression in the form of leptomeningeal enhancement along the cerebellum, although the residual tumor enhancement in the left temporal lobe remained unchanged. Therefore, dabrafenib and trametinib were discontinued.

\section{Patient 2}

Patient 2 was a 24-year-old male with a known left posterior frontal lobe lesion that was discovered after he developed worsening headaches and new-onset seizures. The lesion was non-contrast enhancing and T1-hyperintense, which raised suspicion of a LGG; thus follow-up imaging was recommended. Over the next year, the patient was noncompliant with clinic appointments, surveillance brain MRIs, and antiepileptic medications, resulting in eventual admission for generalized tonic-clonic seizures, word-finding difficulties, and right-sided, upper-extremity weakness. MRI at that time demonstrated an enlarged left posterior frontal lobe lesion with mixed solid and cystic components, with heterogeneously enhancing central foci concerning for progression/transformation of the previously noted lesion. Therefore, the patient underwent a left frontal craniotomy with gross total resection. The postoperative course was complicated by an epidural hematoma and wound dehiscence, requiring debridement and intravenous antibiotics. 
Histopathologic evaluation of the resected tumor showed a variably cellular glioma, with focal areas reminiscent of classic PXA transitioning into more cellular and anaplastic foci akin to epithelioid GBM (Figure 3A). Although PXA areas had somewhat fascicular architecture with pleomorphic tumor cells, intermixed eosinophilic granular bodies (Figure 3B, arrow), chronic inflammatory cells (Figure 3B, asterisk), and xanthic cells (Figure 3C), the anaplastic areas had a much more epithelioid phenotype, analogous to an epithelioid variant of GBM with round, discrete cytoplasmic borders (Figure 3D). Notably, the cells in these latter areas did have an intriguing fine microvesicular cytoplasmic quality. Extensive necrosis (including palisading necrosis), marked endothelial hyperplasia, and numerous mitoses were evident. Foci of increased pericellular reticulin deposition were essentially absent and there was a lack of CD34-positive "spider" cells. Immunostaining for mutant IDH1 R132H was negative. FISH showed polysomy of chromosomes 7 and 10 without EGFR amplification or loss of PTEN (10q). MGMT was unmethylated. BRAF V600E was detected through PCR. These collective findings raised differential possibilities of epithelioid GBM arising in a background of anaplastic
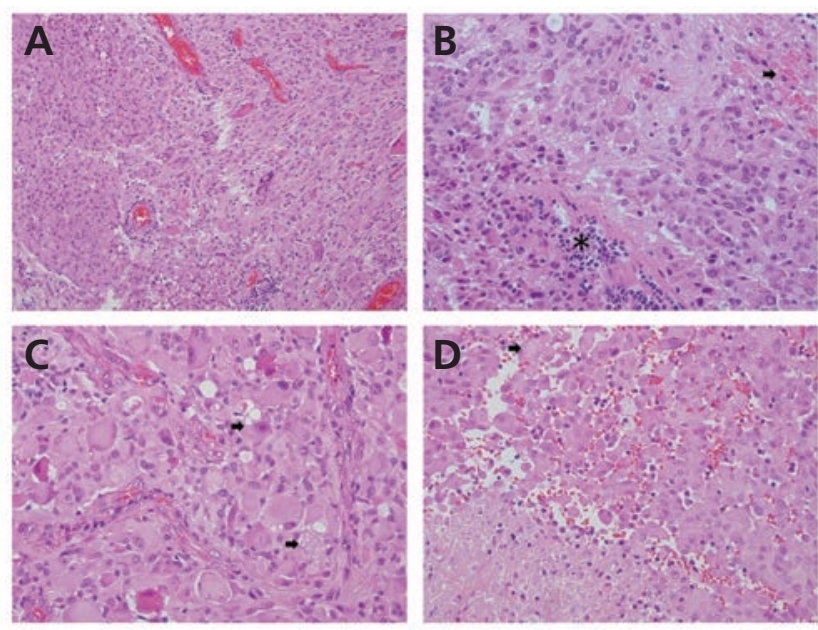

Figure 3. Histopathology of the tumor from patient 2, which has areas akin to epithelioid GBM ( $A$, left) and PXA ( $A$, right; HE staining, original magnification $\times 10)$. Moderately cellular PXA areas are rich in eosinophilic granular bodies (B, arrow) and lymphoplasmacytic cell infiltrate (B, asterisk; HE staining, original magnification $\times 20)$. Xanthic cells are also focally appreciable (C, arrows; HE staining, original magnification $\times 20$ ). Anaplastic foci with large areas of necrosis and epithelioid cell phenotype of tumor cells in areas similar to epithelioid GBM; interestingly, many of the tumor cells have microvesicular quality (D, arrow; HE staining, original magnification $\times 20$ ).

Abbreviations: GBM, glioblastoma; HE, hematoxylin-eosin; PXA, pleomorphic xanthoastrocytoma.
PXA versus an overtly anaplastic PXA. We finally top-lined it as a HGG, with a comment discussing the diagnostic conundrum, and favored the former at the time due to its reminiscence to classic PXA in focal areas.

Whether these are 2 distinct entities or a spectrum of the same remains to be seen, although some recent work suggests an overlap based on the genome-wide methylation analysis. ${ }^{1}$ Interestingly, considerable methylome heterogeneity was noted despite the unifying feature of common BRAF V600E mutations. ${ }^{1}$

After a prolonged recovery, standard-of-care concurrent chemoradiation with temozolomide was initiated and completed without incident. Unfortunately, 1 month later, the patient developed purulent drainage from the incision, requiring multiple washouts, then ultimately underwent removal of the bone flap along with a course of intravenous antibiotics. The initial plan was to complete 6 cycles of high-dose adjuvant temozolomide once antibiotics were completed; however, the patient missed clinic appointments over the subsequent 3 months before re-presenting to the hospital with worsening right-sided weakness. Repeat brain MRI revealed new nodular enhancements along the medial aspect of the resection cavity and pachymeningeal nodular enhancement along the inferior left frontal convexity associated with markedly increased FLAIR signal involving the corticospinal tract, consistent with disease progression. The antiangiogenic agent bevacizumab was started in an attempt to reduce the FLAIR signal and preserve motor function.

Over the next 2 months, the patient had multiple admissions for seizures due to medication noncompliance, delaying the initiation of adjuvant temozolomide, although he continued to receive bevacizumab every 2 weeks. The patient did eventually complete 2 cycles of adjuvant temozolomide, although he continued to be noncompliant with antiepileptic drug treatment, resulting in multiple hospitalizations for seizures. Unfortunately, brain MRI after 2 cycles of temozolomide showed progression of the pachymeningeal-based nodular mass along the left frontal convexity (Figure 4A, white arrow). Therefore, dabrafenib (150 mg, twice daily) and trametinib (2 mg, daily) were started while continuing bevacizumab. Remarkably, the patient presented to clinic for follow-up 1 week later and was no longer 


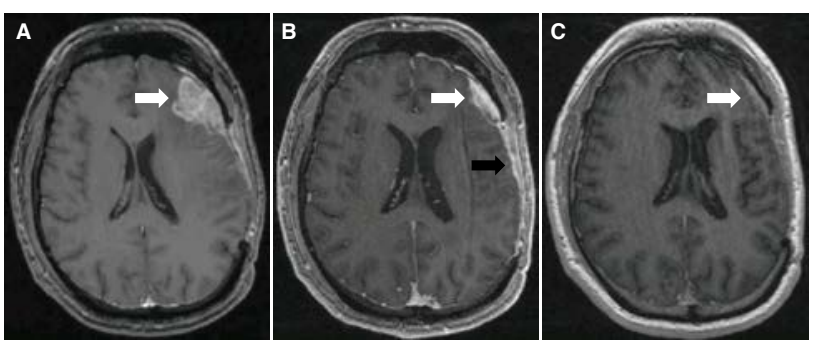

Figure 4. Brain MRI of patient 2 before (A), 4 weeks after (B), and 3 months after (C) treatment with dabrafenib and trametinib. Images are representative axial views from the postcontrast T2-weighted, fluid-attenuated inversion recovery (FLAIR) sequences. The white arrow marks a pachymeningeal-based left frontal lobe lesion. The black arrow indicates area of leptomeningeal gliomatosis, which is resolved on subsequent brain MRI (C).

wheelchair bound, walking independently, with resolving aphasia consistent with a profound clinical response. Consistently, MRI performed 1 month after starting dual-targeted therapy revealed partial regression of the left frontal mass (Figure $4 \mathrm{~B}$, white arrow), although increased leptomeningeal enhancement concerning for leptomeningeal gliomatosis was observed (Figure 4B, black arrow), consistent with a mixed response. Regardless, he continued to improve clinically, and follow-up MRI at 3 months demonstrated continued regression of the left frontal mass and improvement in noted leptomeningeal gliomatosis and vasogenic edema (Figure 4C, white arrow). The patient experienced no breakthrough seizures after starting targeted therapy, and thus bevacizumab was discontinued.

Unfortunately, shortly thereafter the patient again became noncompliant with dabrafenib and trametinib, resulting in rapid disease progression and increased vasogenic edema. After a prolonged hospitalization for status epilepticus, the patient declined further treatment and passed away shortly thereafter.

\section{Discussion}

Unlike melanoma and thyroid cancer, in which somatic BRAF V600E point mutations are present in at least half of cases, ${ }^{2-4}$ their reported frequency in adult HGGs $(1 \%-3 \%)^{5-8}$ is comparable to that of other solid tumor types, such as colorectal carcinoma (3\%), ${ }^{9}$ NSCLC (3\%), ${ }^{10}$ ovarian carcinoma $(0.5 \%),{ }^{11}$ and squamous cell carcinoma of the head and neck $(1.4 \%-3 \%) .{ }^{12,13}$ However, it is important to note that BRAF mutations are enriched in some variants of GBM, such as epithelioid GBM, a rare but recently recognized variant of $I D H$ wild-type GBMs. ${ }^{14}$ Although epithelioid GBMs often lack the common molecular characteristics of adult GBMs, such as EGFR amplification or PTEN loss, the frequency of BRAF V600E mutations in this subgroup is estimated at $50 \% .^{6,15-18}$ Likewise, BRAF V600E mutations are commonly found in LGGs, such as PXA or ganglioglioma, ${ }^{8,19-22}$ and therefore HGGs arising via malignant transformation from these lesions also possess an increased number of $B R A F$ mutations. ${ }^{23-27}$ Interestingly, in some circumstances, the presence of a BRAF mutation may actually drive the transformative process, because BRAF V600E mutations appear to be mutually exclusive with IDH mutations, suggesting that they may be an alternative oncogenic driver in a subset of IDH wild-type LGGs. ${ }^{8,20,22,23,25,28-32}$ However, it is important to note that conclusions from these studies are limited by small sample sizes, although these observations do suggest that patients with GBM, particularly those with GBMs with an epithelioid histology or IDH wild-type secondary GBMs, should be routinely screened for the presence of an actionable BRAF V600E mutation.

To date, with the exception of one case of pediatric GBM, ${ }^{16}$ most case reports describing the successful treatment of BRAF V600E-positive gliomas with BRAF-targeted therapy involve patients with LGGs $^{16,28,33-42}$ (Table 1). As such, the role of targeted therapy in adult BRAF-mutated HGGs has not been explored. Additionally, all previous reports have been in patients with recurrent or refractory disease following standard radiation and cytotoxic chemotherapy, so the role of targeted therapy in newly diagnosed patients is unclear. As such, this is the first report describing the successful treatment of an adult with a BRAF V600E-positive HGG using dual-targeted therapy. Furthermore, we demonstrate for the first time that targeted therapy can safely induce objective and durable clinical responses when used as a first-line agent in patients who are not candidates for aggressive multimodality treatment. Overall, these findings greatly expand on the potential role for these agents in the treatment of BRAF-mutated HGGs.

In metastatic melanoma, dual-targeted therapy with combined BRAF and MEK inhibition has been shown to reduce the rate of secondary skin cancers 
Targeted Therapy in BRAF-Mutated GBM

and the development of resistance. ${ }^{43,44}$ In gliomas, previous studies of targeted therapy have mostly been performed using single-agent BRAF inhibitors ( $\mathrm{Ta}$ ble 1). Thus, the potential benefit of adding an MEK inhibitor (trametinib or cobimetinib) to targeted therapy had not been evaluated in HGGs. We reasoned that combined dabrafenib and trametinib may be more advantageous given the aggressive nature of the disease. Indeed, both reported patients experienced rapid clinical and radiographic improvement. Although the long-term outcome of dual-targeted therapy could not be evaluated in patient 2 due to treatment noncompliance, patient 1 experienced disease control for approximately 11 months before developing progressive disease, which is consistent with results from phase III studies using these agents in metastatic melanoma. ${ }^{43,44}$ These observations suggest that dual-targeted therapy may be an effective therapeutic option for patients with BRAF-mutated HGGs, including as first-line therapy or if a rapid response is needed. Unfortunately, given the rarity of this patient population, larger clinical trials directly comparing the efficacy of dual-targeted therapy with standard-of-care chemoradiation may not be feasible. Therefore, the decision regarding when to use targeted therapy will need to continue to be made on a case-by-case basis for the immediate future.

\section{Conclusions}

This report describes 2 adult patients with BRAF V600E-positive HGG successfully treated with combined dabrafenib and trametinib therapy. Both patients had significant clinical and radiographic responses, consistent with prior results using singleagent BRAF inhibitors in LGGs. Furthermore, these cases propose several important considerations: (1) routine screening for the presence of BRAF point mutations in adult HGGs should be part of the initial molecular characterization; (2) treatment with targeted therapy in newly diagnosed, BRAF-mutated HGGs is a safe and effective approach, particularly in patients deemed to be poor candidates for aggressive chemoradiation; and (3) combination dabrafenib and trametinib remains safe and efficacious for the treatment of central nervous system disease, even when used with antiangiogenic agents. Although these conclusions need to be validated in larger patient cohorts, the case reports herein provide encouraging proof-of-principle data supporting the pursuit of such studies.

\section{References}

1. Alexandrescu S, Korshunov A, Lai SH, et al. Epithelioid glioblastomas and anaplastic epithelioid pleomorphic xanthoastrocytomas-same entity or first cousins? Brain Pathol 2016;26:215-223.

2. Cancer Genome Atlas Network. Genomic classification of cutaneous melanoma. Cell 2015;161:1681-1696.

3. Cancer Genome Atlas Research Network. Integrated genomic characterization of papillary thyroid carcinoma. Cell 2014;159:676-690.

4. Landa I, Ibrahimpasic T, Boucai L, et al. Genomic and transcriptomic hallmarks of poorly differentiated and anaplastic thyroid cancers. J Clin Invest 2016;126:1052-1066.

5. Brennan CW, Verhaak RG, McKenna A, et al. The somatic genomic landscape of glioblastoma. Cell 2013;155:462-477.

6. Behling F, Barrantes-Freer A, Skardelly M, et al. Frequency of BRAF V600E mutations in 969 central nervous system neoplasms. Diagn Pathol 2016;11:55

7. Basto D, Trovisco V, Lopes JM, et al. Mutation analysis of B-RAF gene in human gliomas. Acta Neuropathol 2005;109:207-210.

8. Schindler G, Capper D, Meyer J, et al. Analysis of BRAF V600E mutation in 1,320 nervous system tumors reveals high mutation frequencies in pleomorphic xanthoastrocytoma, ganglioglioma and extra-cerebellar pilocytic astrocytoma. Acta Neuropathol 2011;121:397-405.

9. Cancer Genome Atlas Network. Comprehensive molecular characterization of human colon and rectal cancer. Nature 2012;487:330337.

10. Chen D, Zhang LQ, Huang JF, et al. BRAF mutations in patients with non-small cell lung cancer: a systematic review and meta-analysis. PLoS One 2014;9:e101354.

11. Cancer Genome Atlas Research Network. Integrated genomic analyses of ovarian carcinoma. Nature 2011;474:609-615.

12. Cancer Genome Atlas Network. Comprehensive genomic characterization of head and neck squamous cell carcinomas. Nature 2015;517:576-582.

13. Pickering CR, Zhang J, Yoo SY, et al. Integrative genomic characterization of oral squamous cell carcinoma identifies frequent somatic drivers. Cancer Discov 2013;3:770-781.

14. Louis DN, Perry A, Reifenberger G, et al. The 2016 World Health Organization classification of tumors of the central nervous system: a summary. Acta Neuropathol 2016;131:803-820.

15. Kleinschmidt-DeMasters BK, Aisner DL, Birks DK, Foreman NK. Epithelioid GBMs show a high percentage of BRAF V600E mutation. Am J Surg Pathol 2013;37:685-698.

16. Robinson GW, Orr BA, Gajjar A. Complete clinical regression of a BRAF V600E-mutant pediatric glioblastoma multiforme after BRAF inhibitor therapy. BMC Cancer 2014;14:258.

17. Kleinschmidt-DeMasters BK, Aisner DL, Foreman NK. BRAF VE1 immunoreactivity patterns in epithelioid glioblastomas positive for BRAF V600E mutation. Am J Surg Pathol 2015;39:528-540.

18. Chi AS, Batchelor TT, Yang D, et al. BRAF V600E mutation identifies a subset of low-grade diffusely infiltrating gliomas in adults. J Clin Oncol 2013;31:e233-236.

19. Lee D, Cho YH, Kang SY, et al. BRAF V600E mutations are frequent in dysembryoplastic neuroepithelial tumors and subependymal giant cell astrocytomas. J Surg Oncol 2015;111:359-364.

20. Chappe C, Padovani L, Scavarda D, et al. Dysembryoplastic neuroepithelial tumors share with pleomorphic xanthoastrocytomas and gangliogliomas BRAF(V600E) mutation and expression. Brain Pathol 2013;23:574-583.

21. Dahiya $S$, Haydon $D H$, Alvarado $D$, et al. BRAF(V600E) mutation is a negative prognosticator in pediatric ganglioglioma. Acta Neuropathol 2013;125:901-910. 
Johanns et al

22. Dougherty MJ, Santi M, Brose MS, et al. Activating mutations in BRAF characterize a spectrum of pediatric low-grade gliomas. Neuro Oncol 2010;12:621-630.

23. Matsumura N, Nakajima N, Yamazaki T, et al. Concurrent TERT promoter and BRAF V600E mutation in epithelioid glioblastoma and concomitant low-grade astrocytoma. Neuropathology 2017;37:58-63.

24. Tanaka S, Nakada M, Nobusawa S, et al. Epithelioid glioblastoma arising from pleomorphic xanthoastrocytoma with the BRAF V600E mutation. Brain Tumor Pathol 2014;31:172-176.

25. Nobusawa $S$, Hirato J, Kurihara $H$, et al. Intratumoral heterogeneity of genomic imbalance in a case of epithelioid glioblastoma with BRAF V600E mutation. Brain Pathol 2014;24:239-246.

26. Kuroda J, Nobusawa S, Nakamura H, et al. A case of an epithelioid glioblastoma with the BRAF V600E mutation colocalized with BRAF intact low-grade diffuse astrocytoma. Neuropathology 2016;36:181-186.

27. Funata N, Nobusawa S, Yamada R, Shinoura N. A case of osteoclast-like giant cell-rich epithelioid glioblastoma with BRAF V600E mutation. Brain Tumor Pathol 2016;33:57-62.

28. Chamberlain MC. Salvage therapy with BRAF inhibitors for recurrent pleomorphic xanthoastrocytoma: a retrospective case series. J Neurooncol 2013;114:237-240.

29. Dahiya S, Emnett RJ, Haydon DH, et al. BRAF-V600E mutation in pediatric and adult glioblastoma. Neuro Oncol 2014;16:318-319.

30. Gierke M, Sperveslage J, Schwab D, et al. Analysis of IDH1-R132 mutation, BRAF V600 mutation and KIAA1549-BRAF fusion transcript status in central nervous system tumors supports pediatric tumor classification. J Cancer Res Clin Oncol 2016;142:89-100.

31. Korshunov A, Meyer J, Capper D, et al. Combined molecular analysis of BRAF and IDH1 distinguishes pilocytic astrocytoma from diffuse astrocytoma. Acta Neuropathol 2009;118:401-405.

32. Korshunov A, Ryzhova M, Hovestadt V, et al. Integrated analysis of pediatric glioblastoma reveals a subset of biologically favorable tumors with associated molecular prognostic markers. Acta Neuropathol 2015;129:669-678.

33. Bautista F, Paci A, Minard-Colin V, et al. Vemurafenib in pediatric patients with BRAFV600E mutated high-grade gliomas. Pediatr Blood Cancer 2014;61:1101-1103.
34. Rush S, Foreman N, Liu A. Brainstem ganglioglioma successfully treated with vemurafenib. J Clin Oncol 2013;31:e159-160.

35. Usubalieva A, Pierson CR, Kavran CA, et al. Primary meningeal pleomorphic xanthoastrocytoma with anaplastic features: a report of 2 cases, one with BRAF(V600E) mutation and clinical response to the BRAF inhibitor dabrafenib. J Neuropathol Exp Neurol 2015;74:960-969.

36. Skrypek M, Foreman N, Guillaume D, Moertel C. Pilomyxoid astrocytoma treated successfully with vemurafenib. Pediatr Blood Cancer 2014;61:2099-2100.

37. Dabrafenib effective in pediatric glioma. Cancer Discov 2017;7:OF5.

38. Aguilera D, Janss A, Mazewski C, et al. Successful retreatment of a child with a refractory brainstem ganglioglioma with vemurafenib. Pediatr Blood Cancer 2016;63:541-543.

39. del Bufalo F, Carai A, Figa-Talamanca L, et al. Response of recurrent BRAFV600E mutated ganglioglioma to vemurafenib as single agent. J Transl Med 2014;12:356.

40. Lassaletta A, Guerreiro Stucklin A, Ramaswamy V, et al. Profound clinical and radiological response to BRAF inhibition in a 2-month-old diencephalic child with hypothalamic/chiasmatic glioma. Pediatr Blood Cancer 2016;63:2038-2041.

41. Meletath SK, Pavlick D, Brennan $T$, et al. Personalized treatment for a patient with a BRAF V600E mutation using dabrafenib and a tumor treatment fields device in a high-grade glioma arising from ganglioglioma. J Natl Compr Canc Netw 2016;14:1345-1350.

42. Lee EQ, Ruland S, LeBoeuf NR, et al. Successful treatment of a progressive BRAF V600E-mutated anaplastic pleomorphic xanthoastrocytoma with vemurafenib monotherapy. J Clin Oncol 2016;34:e87-89.

43. Long GV, Stroyakovskiy D, Gogas H, et al. Dabrafenib and trametinib versus dabrafenib and placebo for Val600 BRAF-mutant melanoma: a multicentre, double-blind, phase 3 randomised controlled trial. Lancet 2015;386:444-451.

44. Larkin J, Ascierto PA, Dreno B, et al. Combined vemurafenib and cobimetinib in BRAF-mutated melanoma. N Engl J Med 2014;371:18671876. 